\title{
A Comparative Study of E-Learning Technique with Traditional Teaching Techniques
}

\author{
Sunita Gaur ${ }^{1}$, Archana Chaudhary ${ }^{2}$, Mithilesh Mittal $^{3}$ \\ Lecturer, School of Computer Science \& IT, Devi Ahilya Vishwa Vidyalaya, Indore, India ${ }^{1}$ \\ Senior Lecturer, School of Computer Science \& IT, Devi Ahilya Vishwa Vidyalaya, Indore, India ${ }^{2}$ \\ Professor, Govt. Holkar Science College, Indore, India ${ }^{3}$
}

\begin{abstract}
E-learning System has been an increasing focus as a vital teaching method for more than a decade. This paper focuses upon a comparative study of E-learning teaching techniques with traditional teaching techniques. The feedback from different user groups -students, researchers, teachers and staff is considered for traditional teaching techniques and e-learning based teaching techniques. The tools used showed the effectiveness of e-learning techniques over traditional teaching methods. The study showed that e-learning techniques attained high positive feedback for elearning techniques as compared to traditional teaching methods for all the user categories.
\end{abstract}

Keywords: E-learning, Web Guru, Traditional learning.

\section{INTRODUCTION}

In recent times, there have been advances in the development of software technology in particular in the field of education [1]. Combined learning strategies can optimise the integration of multi-modal, multi-channel and multi-source learning which includes online and traditional learning; this helps learners develop and improve their learning independence and to self-manage to best suit their education style, the way of work and working style. Such software applications are generally developed on web 2.0 tools, for example mobile-learning applications, twitter, YouTube, slide share, Picasa, media wiki, etc.

In the field of education this software is used to help teachers to monitor students' activities. In particular terms, e-learning is based not only on distributed learning, online learning, virtual learning, web based training or networked learning but also on testing and evaluating the best feedback, involvement and the interaction of some platforms in e-learning environments between the instructor and learner [2]. Interest in e-learning is on an increasing development mainly for those already in fulltime employment and dedicated to continue their education and/or professional training. The percentage of companies planning to provide e-learning support for their staff has risen from $38.5 \%$ in 2007 to $51 \%$ in 2011 [3]. The e-learning system notion includes learning content, but also the infrastructure that allows content to be created, stored, accessed, and delivered and the learner and the learning process to be managed. The e-learning is the unifying term to describe the fields of online learning, elearning is simply using technology to deliver web based training and educational materials. Although many students have strong opinions about where e-learning is likely to go and how quickly it may or may not get there.

Some formal definitions of e-learning are-

- The delivery of a learning, training or education program by electronic device. E-learning involves the use of a computer system or electronic device (e.g. a mobile phone) in some way to give web based training, educational or study material.

- The convergence of the internet and e-learning, or internet-enabled learning.

- Application of network technology to create foster, deliver and facilitate learning, anytime and anywhere.

- The release of individualized, whole, dynamic learning content in real time, aiding the development of communities of knowledge, linking learners and practitioners with experts.

- An observable fact delivering responsibility, openness, and opportunity to allow people and organization to keep up with the rapid changes that define the internet world.

- A force that gives public and organizations the competitive edge to allow them to keep ahead of the rapidly changing global economy [4].

The Learning Management System (LMS) does not only provide the facility to the instructors for assessment and the measurement of the performance of the students in an interactive learning process [5]. This paper reviews Monitoring and Analysis Tool for the e-learning Program (WebGuru) which is used in area of e-learning in Devi Ahilya University for improving teaching learning skills. The paper is also supported with an analysis of e-learning with WebGuru and traditional teaching methods viz. Black Board teaching, Topic explanation, student understanding, class room interaction etc. The results of experiments conducted with different types of groups - students, research scholars, teachers and other staff are also showed in this work. This paper is organized as: section 2 gives literature review. Section 3 presents results and discussion and section 4 finally describes conclusion drawn.

\section{LITERATURE REVIEW}

E-learning networked information and communications technology is a modern process in the teaching and learning of undergraduate and postgraduate students. There are a number of provisions that are used for the 
purpose of describing this online mode of teaching and elearning. The technique most widely uses online activities, virtual learning, and a web based e-learning network and distributed teaching [4]. In this type of learning delivery, information and communications technology are followed in synchronous and asynchronous modes.

It is clear from the information available, the role of communication and information technology is to provide opportunities in the areas of storing, capturing and distributing information [6]. With the event of media software tools and equipments, radio and television are areas that could be used for debate [7]. Video clips used for role-play based learning have made the learning and teaching experience more appealing and revolutionary [6]. It has been seen that students who generally want to stay away from school are highly motivated by innovative teaching methods.

Moving images and educational instructions given through multi media have aided in increasing the ability of instructors to represent information, in many ways within the school or college [7]. With the popularity of radio and television and such other media, it is assumed that every media type has the capability and potential to increase, direct, and shape individual's abilities to communicate and instruct. Computers are second in line to provide technological innovation, both with the help of the latest software and hardware, in the area of valuable e-learning process. Though, computers have been used for learning purposes since the 1960s and 70s they were taken up more enthusiastically among e-learning volunteers, students and teachers during 2000 with the help of the Internet [8]. However, there are many researchers who are of the opinion that the impact of computer technology on learning is less than its economic benefits and in some cases they did not show any benefits on learning. Most of the researchers are of the opinion that learning is acquired not with improvement in technology alone but with the content presented in the medium.

\section{RESULTS AND DISCUSSION}

Web guru software and framework that are used to perform e-learning will be evaluated on the criterion of the personal experience of instructors and learners. The teacher's concern that they are not able to trace the learner's activity while their entire teaching session and the learner's question about the validity and reliability of the content delivered in the e-learning process $[9,10]$. The experiment makes use of samples of a few instructors and e-learners that are using Web guru software tool as their virtual framework for e-learning. A questionnaire was prepared in order to get the responses made by the sample user group.

The questionnaire was distributed to user groups that involved students, researchers, teachers and other staff members, both male and female gender of different ages.

The questions were based on the pattern of direct asking. There were questions based upon either 'Yes' or 'No' responses. Questions were the same for all the respondents and the language was used as English. On the basis of questionnaires some important results are discussed below. The graphs below show that e-learning techniques are first choice of all the user groups as compared to traditional teaching methods.

For conceptual understanding also e-learning techniques were first choice for all user groups:

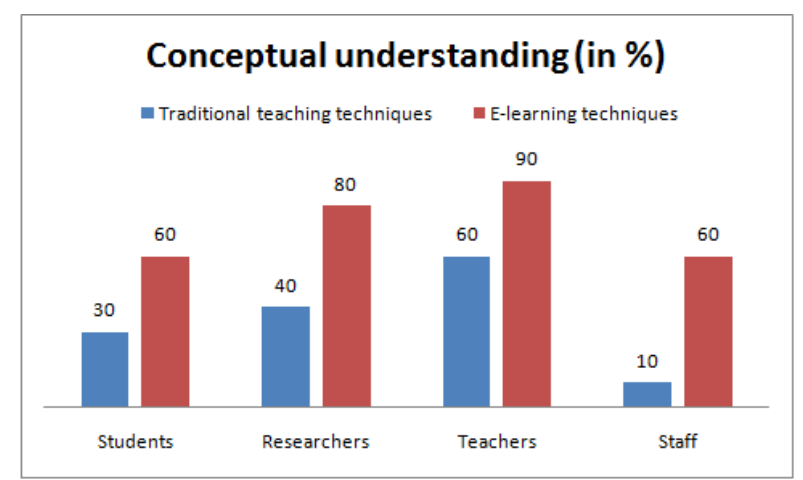

Fig. 1. Graph showing conceptual understanding for both learning techniques

Discussion: This is a question that was asked to all user groups -student, researcher, teacher and staff that conceptual understanding is enhanced by traditional teaching techniques or e-learning based teaching techniques. As the graph indicates, from the four groups: in case of students $60 \%$ conceptual understanding is developed by e-learning techniques whereas only $30 \%$ of conceptual understanding is developed using traditional teaching techniques. Similarly other groups also offered more points to conceptual understanding through elearning techniques as compared to traditional techniques.

One important learning parameter was taken as actual learning. The graph below shows how different user categories voted for actual learning with respect to elearning techniques as well as traditional teaching techniques.

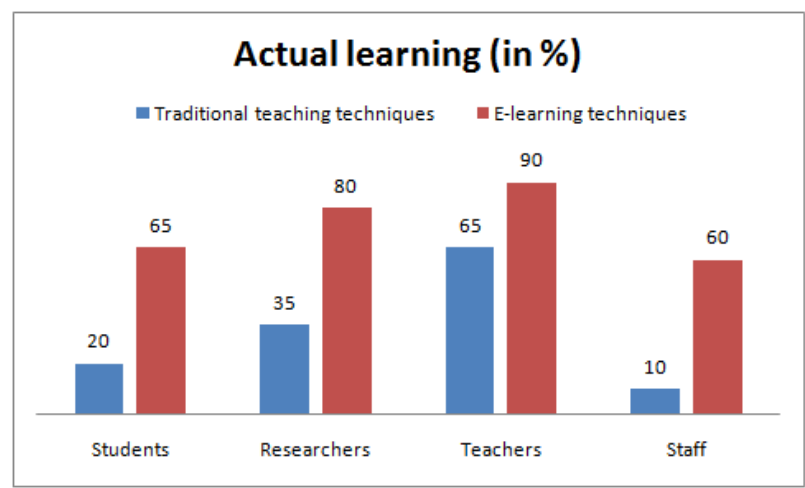

Fig. 2. Graph showing actual learning ability with Elearning techniques and traditional teaching techniques

Discussion: The question about what was the actual learning of a subject or topic with the two types of techniques in the context was asked to all user groups student, researcher, teacher and staff. As the graph above indicates, in case of students $65 \%$ of actual learning is 
developed by e-learning techniques whereas only $20 \%$ of actual learning is developed using traditional teaching techniques. Similarly other groups also offered more appreciation to e-learning techniques for actual understanding as compared to traditional techniques.

\section{CONCLUSION}

The study made showed the effectiveness of e-learning techniques over traditional teaching methods. The study showed that e-learning techniques attained high response as compared to traditional teaching methods for all the user categories. Finally feedback of traditional teaching techniques and e-learning techniques from all user categories is shown by the graph below.

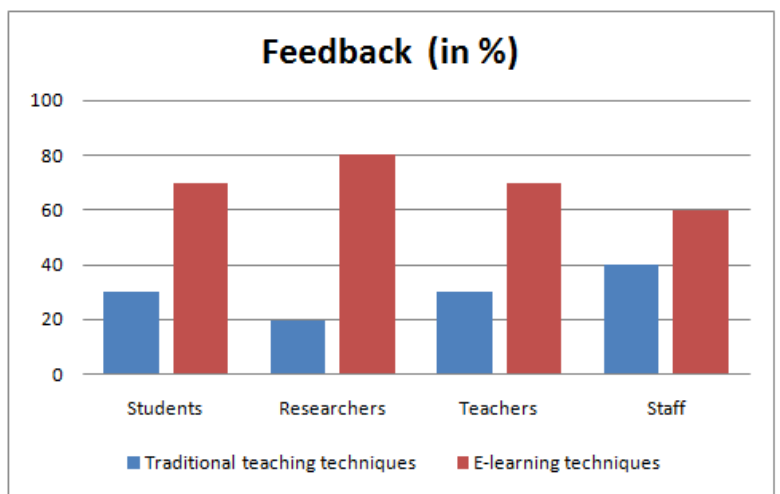

Fig.3. Graph showing the feedback of E-learning techniques and traditional teaching techniques

It is clear from the graph above that e-learning techniques have good feedback from students, researchers, teachers and staff members. It is clear from the above graph that $70 \%$ of students gave positive feedback for e-learning techniques and only $30 \%$ of students preferred traditional methods of teaching. Similarly $80 \%$ of researchers preferred e-learning and only $20 \%$ of researchers gave positive feedback for traditional methods. It is also clear from the graph above that $70 \%$ of teachers appreciate elearning and only $30 \%$ of teachers appreciate traditional teaching methods. In case of staff members $70 \%$ of staff members gave positive feedback for e-learning techniques and $30 \%$ gave positive feedback for traditional teaching techniques.

\section{REFERENCES}

[1] S. M. Metev and V. P. Veiko, Laser Assisted Microtechnology, 2nd ed., R. M. Osgood, Jr., Ed. Berlin, Germany: Springer-Verlag, 1998.

[2] J. Breckling, Ed., The Analysis of Directional Time Series: Applications to Wind Speed and Direction, ser. Lecture Notes in Statistics. Berlin, Germany: Springer, 1989, vol. 61.

[2] S. Zhang, C. Zhu, J. K. O. Sin, and P. K. T. Mok, "A novel ultrathin elevated channel low-temperature poly-Si TFT," IEEE Electron Device Lett., vol. 20, pp. 569-571, Nov. 1999.

[3] M. Wegmuller, J. P. von der Weid, P. Oberson, and N. Gisin, "High resolution fiber distributed measurements with coherent OFDR," in Proc. ECOC'00, 2000, paper 11.3.4, p. 109.

[4] R. E. Sorace, V. S. Reinhardt, and S. A. Vaughn, "High-speed digital-to-RF converter," U.S. Patent 5668 842, Sept. 16, 1997.

[5] (2002) The IEEE website. [Online]. Available: http://www.ieee.org/
6] M. Shell. (2002) IEEEtran homepage on CTAN. [Online]. Available: http://www.ctan.org/tex-archive/macros/ latex /contrib. /supported/IEEEtran/

[7] FLEXChip Signal Processor (MC68175/D), Motorola, 1996

[8] "PDCA12-70 data sheet," Opto Speed SA, Mezzovico, Switzerland.

[9] A. Karnik, "Performance of TCP congestion control with rate feedback: TCP/ABR and rate adaptive TCP/IP," M. Eng. thesis, Indian Institute of Science, Bangalore, India, Jan. 1999.

[10] J. Padhye, V. Firoiu, and D. Towsley, "A stochastic model of TCP Reno congestion avoidance and control," Univ. of Massachusetts, Amherst, MA, CMPSCI Tech. Rep. 99-02, 1999.

[11] Wireless LAN Medium Access Control (MAC) and Physical Layer (PHY) Specification, IEEE Std. 802.11, 1997. 